\title{
Learning a Two-Dimensional Fuzzy Discriminant Locality Preserving Subspace for Visual Recognition
}

\author{
Ruicong $\mathrm{ZHI}^{\dagger, \dagger \dagger \mathrm{a})}$, Member, Lei $\mathrm{ZHAO}^{\dagger \dagger}$, Bolin $\mathrm{SHI}^{\dagger \dagger}$, and $\mathrm{Yi} \mathrm{JIN}^{\dagger}$, Nonmembers
}

SUMMARY A novel Two-dimensional Fuzzy Discriminant Locality Preserving Projections (2D-FDLPP) algorithm is proposed for learning effective subspace of two-dimensional images. The 2D-FDLPP algorithm is derived from the Two-dimensional Locality Preserving Projections (2DLPP) by exploiting both fuzzy and discriminant properties. 2D-FDLPP algorithm preserves the relationship degree of each sample belonging to given classes with fuzzy $k$-nearest neighbor classifier. Also, it introduces between-class scatter constrain and label information into 2D-LPP algorithm. 2D-FDLPP algorithm finds the subspace which can best discriminate different pattern classes and weakens the environment factors according to soft assignment method. Therefore, 2D-FDLPP algorithm has more discriminant power than 2D-LPP, and is more suitable for recognition tasks. Experiments are conducted on the MNIST database for handwritten image classification, the JAFFE database and Cohn-Kanade database for facial expression recognition and the ORL database for face recognition. Experimental results reported the effectiveness of our proposed algorithm.

key words: fuzzy assignment, discriminant objective function, locality preserving, pattern recognition

\section{Introduction}

Learning effective data representations are one of the most important issues in pattern recognition. Researchers showed that the intrinsic characterization of data always lied on the corresponding feature subspace. For image-based recognition tasks, for example, face recognition [1], facial expression recognition [2], object pose classification [3] and other pattern recognition tasks [4]-[6], it is essential to find effective features in the image-based subspace. In many real world applications, one often faces to the curse of dimensionality problem [7] which arises from the fact that there are usually too few samples comparing with the number of dimensions. To overcome the curse of dimensionality, researchers made effort to find low-dimensional representation for original images and made a growing interest in subspace analysis techniques.

From a geometrical point of view, the dimensionality reduction can be formulated as discovering a lowdimensional embedding of high-dimensional data, which is assumed to lie on a linear or nonlinear manifold. There are many subspace-based methods which can be divided into two categories: linear (e.g. PCA, LDA, LPP, ICA,

Manuscript received November 27, 2013.

Manuscript revised May 9, 2014.

${ }^{\dagger}$ The authors are with the School of Computer \& Information Technology, Beijing Jiaotong University, Beijing 100044, China.

${ }^{\dagger}$ The authors are with the Institute of Food and Agricultural Standardization, China National Institute of Standardization, Beijing 100191, China.

a) E-mail: zhirc_research@ @otmail.com

DOI: $10.1587 /$ transinf.2013EDP7422
NMF, etc.) [8]-[10] and nonlinear (e.g. kernel-based algorithms, manifold learning algorithms and spectral based algorithms) [11]-[13]. The differences between these methods depend on their different motivations and objective functions. The tree illustration of the main subspace-based algorithms is shown in Fig. 1. The linear algorithms include vector-based algorithms and matrix-based algorithms. Vector-based algorithms treat images as vectors, and twodimensional (2D) image matrices must be previously transformed into one-dimensional (1D) image vectors which usually lead to a high-dimensional image vector space. Therefore, it is difficult to evaluate the resulting objective matrix accurately due to its large size and the relatively small number of training samples (or small sample size problem). Matrix-based algorithms are straightforward image projection techniques, which treat the image matrices directly according to various objective functions. They successfully solve the small sample size problem and evaluate the image objective matrices accurately [14], [15]. Furthermore, less time is required for calculation. It is reported that matrixbased algorithms outperform vector-based algorithms significantly on both recognition accuracies and computation time.

Two-dimensional Locality Preserving Projections (2DLPP) [16] is a recent proposed algorithm which can obtain a linear subspace that preserves local information of the image set. The locality characterization is likely a nearest neighbor searching in the low-dimensional space, and it will yield similar results to that in the high dimensional space [17]. A good many of experiments show that 2D-LPP has more discriminating power than Two-dimensional Principal Component Analysis (2D-PCA) and Two-dimensional Linear Dis-

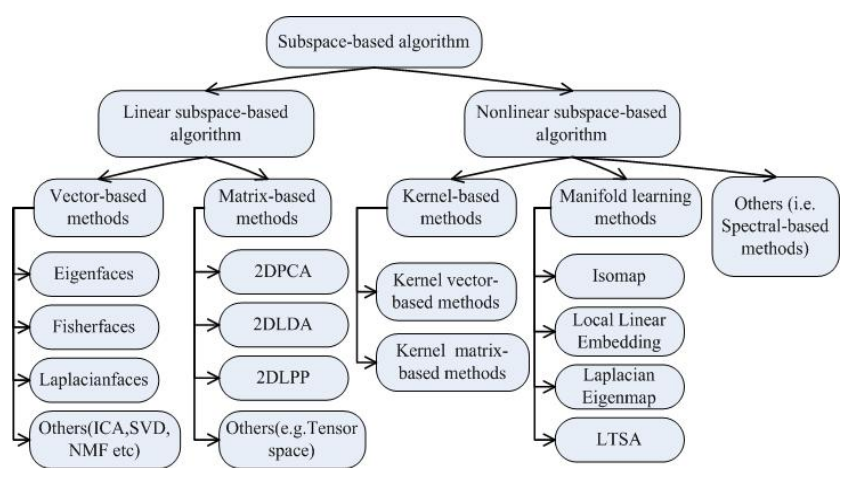

Fig. 1 The main subspace-based algorithms. 
criminant Analysis (2D-LDA) [16], [18].

2D-LPP has been successfully applied to biometrics recognition, e.g. face recognition, palmprint recognition, facial expression recognition. Shan et. al. [12] reported the comprehensive comparison of popular linear subspace methods for facial expression recognition, e.g. PCA, LDA, LPP (supervised and unsupervised), ONPP (Orthogonal Neighborhood Preserving Projections, supervised and unsupervised). It concludes that supervised LPP performs best in supervised methods for facial expression recognition. Moreover, a great number of researchers tried to improve the efficiency of LPP [20]-[22]. However, if the training samples are insufficient and data dimension is high especially for image data, LPP can not be used directly due to singularity of matrices.

Conducting LPP in two-dimensional sense based directly on image matrices, $2 \mathrm{D}$-LPP is proposed. 2D-LPP does not suffer from small sample size problem, and outperforms LPP algorithm on visual recognition problems [16], [19], [23].

However, 2D-LPP suffers from the limitation that it preserves the locality structure of data points, and ignores the locality structure between pattern classes, that means it deemphasizes discriminant information which is very important in pattern recognition tasks. On the other hand, 2DLPP algorithm adopts the binary class assignment, which means that the samples come fully assigned to the given classes. Evidently, visual recognition problem usually affected by numerous conditions, it is advantageous to investigate these factors on soft class assignment. By considering these two aspects, we propose a novel Two-dimensional Fuzzy Discriminant Locality Preserving Projections (2DFDLPP) to learn a more effective subspace for image representation. 2D-FDLPP is a linear dimensionality reduction method, and it is derived by introducing discriminant information to 2D-LPP algorithm. Moreover, 2D-FDLPP utilizes a fuzzy assignment method to determine the coefficients of weight matrix.

The technique of fuzzy set has been successfully used in image segmentation, image filtering, objection detection and pattern recognition. It is usually done by coping with the factor of uncertainty being inherently presented in image processing and pattern recognition. From this point of view, we address the uncertainty assignment to the weight coefficients so that to get fuzzy weight matrix. The idea of fuzzification of class assignment can be dated back to the results published by Keller et. al. [24] coming under the notion of a fuzzy $k$-nearest neighbor classifier. The modification makes the new method be more discriminant and robust to the confused images.

The remainder of this paper is organized as follows: Section 2 gives the review of algorithm 2D-LPP; Section 3 analyzes the 2D-FDLPP algorithm in detail; Section 4 reports the experimental results on different databases; conclusions are presented in Sect. 5.

\section{Review of 2D-LPP Algorithm}

2D-LPP works directly on the image matrix, and it is a linear dimensionality reduction method which aims to find a transformation matrix $A$ that maps original image $X_{i}$ to a low-dimensional representation matrix $Y_{i}$, according to the transformation $Y_{i}=X_{i} A$. $X_{i}$ denotes an $h \times w$ original image matrix, $Y_{i}$ is the low-dimensional matrix corresponding to the original image matrix, and $A$ is the transformation matrix.

The linear transformation can be obtained by minimizing the objective function as follows:

$$
\min \sum_{i j}\left\|Y_{i}-Y_{j}\right\|^{2} S_{i j}
$$

The objective function can be reduced to:

$$
\begin{aligned}
J=\frac{1}{2} \sum_{i, j}\left\|Y_{i}-Y_{j}\right\|^{2} S_{i j} & =\frac{1}{2} \sum_{i, j}\left\|X_{i} A-X_{j} A\right\|^{2} S_{i j} \\
& =A^{T} X^{T}\left(L \otimes I_{h}\right) X A
\end{aligned}
$$

where $D$ is a diagonal matrix whose entries are column (or row) sum of $S, L=D-S$ is the Laplacian matrix. $X$ is data matrix, $X=\left[X_{1}, X_{2}, \ldots, X_{N}\right]$. It contains $N$ samples which belong to $C$ pattern classes, and each class contains $n_{i}(i=1,2, \ldots, C)$ samples. With the constraint $A^{T} X^{T}(D \otimes$ $\left.I_{h}\right) X A=1$, the optimization problem is reduced to finding:

$$
\min _{A^{T} X^{T}\left(D \otimes I_{h}\right) X A} A^{T} X^{T}\left(L \otimes I_{h}\right) X A
$$

\section{Two-Dimensional Fuzzy Discriminant Locality Pre- serving Projections (2D-FDLPP) Algorithm}

Although 2D-LPP preserves the local structure of the image space, it deemphasizes the locality characters between pattern classes. As discriminant information is very important for pattern recognition problem, we introduce label information and between-class scatter constraint into the objective function of 2D-LPP, so that to exact more discriminating information of images. On the other hand, 2D-LPP algorithm assigns the samples in a crisp way to the given classes. That means, for each sample, it fully belongs to or not belongs to a pattern class. However, pattern recognition problems are easily affected by several factors, e.g. environment factors. It is more suitable to assign the samples in a soft way. Therefore, we propose Two-dimensional Fuzzy Discriminant Locality Preserving Projections (2D-FDLPP). 2D-FDLPP is interesting in the following three aspects: (1) 2D-FDLPP is based on image matrix, and it solves the optimal problem directly, which makes the calculation accurate and less time consumed; (2) 2D-FDLPP utilizes soft assignment method to construct the weight matrix with membership grade of each data point belonging to given class; (3) the betweenclass scatter constraint is added into the 2D-FDLPP objection function and 2D-FDLPP aims to find the subspace by 
minimizing the within-class distance, while maximizing the between-class distance.

\subsection{Fuzzy Weight Matrix}

A number of studies have been carried out to cope with the factor of uncertain being inherently present in many problems of image processing and pattern recognition, and these can be called as fuzzy image processing. Generally speaking, pattern recognition problem is often affected by environment factors, for instance, illumination and pose. By introducing uncertainty associated with the affective factors, one can get more robust data presentation for pattern recognition. From this point of view, we propose a fuzzy class allocation method instead of conventional crisp allocation method. The weight coefficients of pairs of samples are defined by membership degree [25]. Fuzzy $k$-nearest neighbor classifier is used to calculate the degree of membership of each sample belonging to the given class.

As usual, the partition matrix is denoted by $U=\mu_{i j}$, $\mu_{i j}$ denotes the membership grade of sample $j$ belonging to class $i$, and it satisfies two properties:
(a) $\sum_{i=1}^{C} \mu_{i j}=1$
(b) $0<\sum_{j=1}^{N} \mu_{i j}<N$

The larger the value of $\mu_{i j}$, the higher degree of membership that sample $j$ belongs to class $i$. For instance, $\mu_{i j}=1$ denotes that sample $j$ exactly belongs to class $i$, and $\mu_{i j}=0$ denotes that sample $j$ does not belong to class $i$.

In fuzzy weight matrix construction, we try to find a better representation of the class label information of training samples. By taking fuzzy $k$-nearest neighbor classifier on the training samples, we find the first $k$ nearest neighbors of the sample according to the Euclidean distance (the Euclidean distance between sample $i$ and sample $j$ is defined as $\left.d i s_{i j}=\sum_{k, l}\left(X_{k, l}^{i}-X_{k, l}^{j}\right)^{2}\right)$, then collect the number of samples belong to the certain pattern classes that they belong to, i.e. $n_{i j}$ denotes the number of sample $j$ 's neighbor which belongs to class $i$, then the membership degree is calculated according to the neighbor samples' class label. Let $\tilde{S}$ denote the fuzzy weight matrix between training samples, and we use the membership degree of each sample belonging to classes to construct the weight coefficients of $\tilde{S}$. If sample $k$ belongs to class $i$, then the weight value between sample $j$ and sample $k$ is obtained by the degree of membership of sample $j$ belonging to class $i$. $\tilde{D}$ is a diagonal matrix which entries are row sum of $\tilde{S}$.

Therefore, the weight matrix reflects the degree of membership of each sample belonging to given class rather than the relationship between pairs of samples. Each sample is assigned a membership value to each class rather than binary decision of belongs to or does not belong to. The advantage of such assignment is that these membership values act as strength or confidence with which the current sample belongs to a particular class. The details of the procedure of construct the fuzzy Laplacian matrix is shown in Fig. 2.

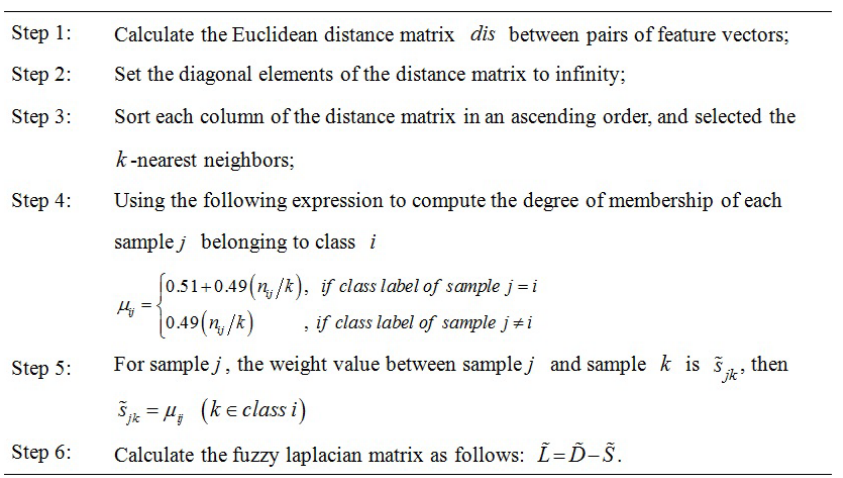

Fig. 2 Main steps of constructing the fuzzy Laplacian matrix.

Anger
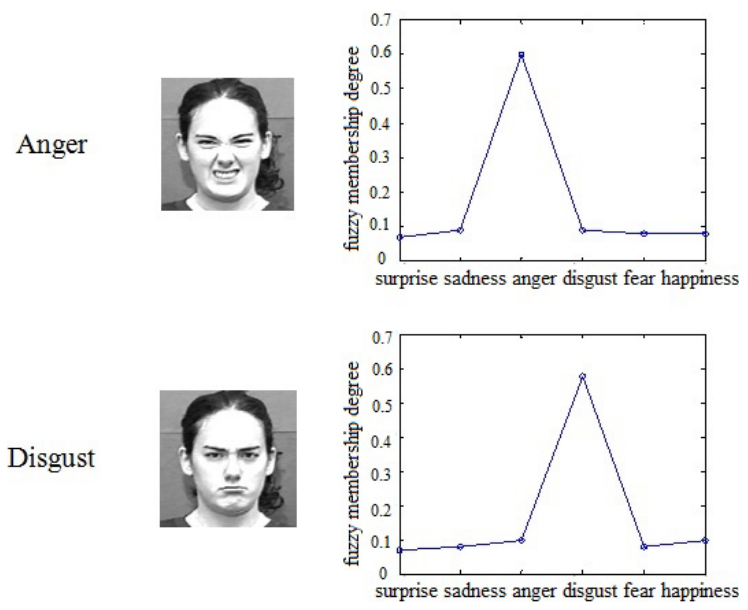

Fig. 3 Membership degree to different classes.

The following example comes from Cohn-Kanade facial expression database. Figure 3 shows the membership degrees obtained by the fuzzy $k$-nearest neighbor classifier. For facial expression anger, the membership degree is 0.6, while membership degrees of other facial expressions are between $0.05-0.1$. The confusing impact of different facial expressions is quantified by the internal membership degree. By this soft assignment, the reflection impacts are weakened and the sample is confirmed to class anger.

\subsection{Two-Dimensional Discriminant Objective Function}

Discriminant information is very important for pattern recognition problem, so we add the between class scatter constraint into the objective function. Therefore, the discriminant subspace can be obtained by minimizing the within-class distance, while maximizing the between-class distance. Therefore, the Two-dimensional discriminant objective function is

$$
J_{D}(Y)=\frac{\sum_{i, j=1}^{N}\left\|Y_{i}-Y_{j}\right\|^{2} S_{i j}}{\sum_{i, j=1}^{C}\left\|M_{i}-M_{j}\right\|^{2} W_{i j}}
$$

where $Y_{i}, Y_{j}$ denote the low-dimensional image matrices, corresponding to the original images. $S$ is the weight matrix 
of the samples, $S_{i j}=\exp \left(-\left\|X_{i}-X_{j}\right\|^{2} / t\right)(t$ is the empirical parameter). $W$ is the weight matrix of classes. $M_{i}$ represents the mean matrix of the projected images in class $i$, i.e. $M_{i}=$ $\left(1 / n_{i}\right) \sum_{k=1}^{n_{i}} Y_{k}^{i}$.

According to the linear transformation $Y_{i}=X_{i} A$, the denominator of the objective function can be reformed as follows:

$$
\begin{aligned}
& \frac{1}{2} \sum_{i, j=1}^{C}\left(M_{i}-M_{j}\right)^{T}\left(M_{i}-M_{j}\right) W_{i j} \\
= & \sum_{i=1}^{C} A^{T} F_{i}^{T} E_{i i} F_{i} A-\sum_{i, j=1}^{C} A^{T} F_{i}^{T} W_{i j} F_{j} A \\
= & A^{T} F^{T}\left((E-W) \otimes I_{h}\right) F A
\end{aligned}
$$

where $F_{i}=\left(1 / n_{i}\right) \sum_{k=1}^{n_{i}} X_{k}^{i}, E$ is a diagonal matrix whose entries are column (or row) sum of $W, H=E-W$. Similarly, the numerator of the objective function is

$$
\begin{aligned}
& \frac{1}{2} \sum_{i, j=1}^{N}\left(Y_{i}-Y_{j}\right)^{T}\left(Y_{i}-Y_{j}\right) S_{i j} \\
= & \sum_{i=1}^{N} A^{T} X_{i}^{T} D_{i i} X_{i} A-\sum_{i, j=1}^{N} A^{T} X_{i}^{T} S_{i j} X_{j} A \\
= & A^{T}\left[\sum_{i, j=1}^{N} X_{i}^{T}\left(D_{i i}-S_{i j}\right) X_{j}\right] A \\
= & A^{T} X^{T}\left(L \otimes I_{h}\right) X A
\end{aligned}
$$

Therefore, the projection directions can be obtained by minimizing the above function

$$
A=\arg \min _{A} \frac{A^{T} X^{T}\left(L \otimes I_{h}\right) X A}{A^{T} F^{T}\left(H \otimes I_{h}\right) F A}
$$

By using Lagrange multipliers, the minimization problem becomes a generalized eigenvalue problem:

$$
X^{T}\left(L \otimes I_{h}\right) X A=\lambda F^{T}\left(H \otimes I_{h}\right) F A
$$

Thus, $A=\left[a_{1}, a_{2}, \ldots, a_{d}\right]$ is $w \times d$ with columns being the eigenvectors corresponding to the $d$ largest eigenvalues. All the images should be embedded according to the linear projection $Y_{i}=X_{i} A$ for classification.

The original between-class scatter matrix overemphasizes the classes with large distances, but pays little attention to the classes with small distances. So the projection directions can preserve the distances of already wellseparated classes but cause a large overlap of neighboring classes. To prevent the overlap problem, we introduce the weighting function $w\left(d_{i}\right)$ into between-class scatter matrix to balance the distances between classes. $d_{i}$ presents the distance between the center of class $i$ and the center of total samples, and $w\left(d_{i}\right)$ is a monotony and descending function corresponding to $d_{i}$. Therefore, more powerful discriminant information can be obtained. In our 2D-FDLPP algorithm, the weight values are chosen as $W_{i j}=\exp \left(-\left\|F_{i}-F_{j}\right\|^{2} / t\right)$ ( $t$ is the empirical parameter).

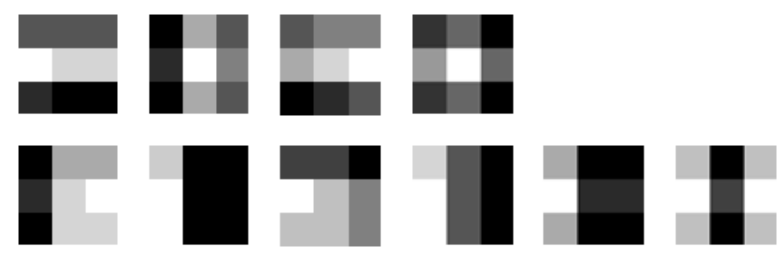

Fig. 4 Ten simulation images.

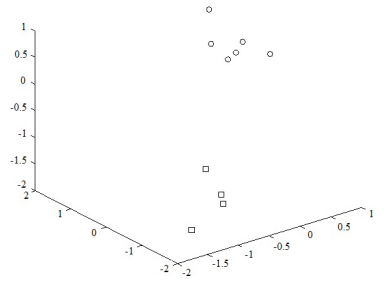

(a) 2D-FDLPP

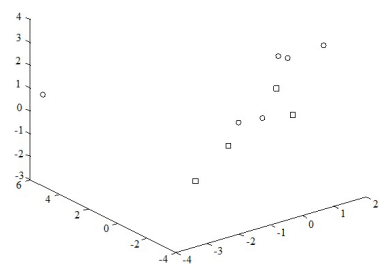

(b)2D-LPP
Fig. 5 Ten simulation images.

\subsection{Learning Two-Dimensional Fuzzy Discriminant Lo- cality Preserving Subspace}

As we can see from the above analysis, we take both of the fuzzy and discriminant characterizations to propose more effective algorithm - Two-dimensional Fuzzy Discriminant Locality Preserving Projections (2D-FDLPP). 2D-FDLPP utilizes the fuzzy assignment to construct the weight matrix, and minimizes the discriminant objective function Eq. (7) to obtain the discriminant optimal projective directions. Therefore, the locality preserving subspace with fuzzy and discriminant characterization can be obtained by solving the generalized eigenvalue problem Eq. (8) with fuzzy weight matrix described in Sect. 3.1.

To demonstrate the improvement of 2D-FDLPP algorithm over 2D-LPP algorithm, we performed a simple simulation example. In our simulations, the data vectors were $3 \times 3$-pixel images of two classes. The images are shown in Fig. 4, and images on the first row belong to class 1 and images on the second row belong to class 2 . The images are projected by 2D-FDLPP algorithm and 2D-LPP algorithm respectively and the projected images are illustrated in a three-dimensional way as shown in Fig. 5.

Figure 5 (a) shows the distribution of the images in three-dimensional 2D-FDLPP subspace, which reflects a good separation between the two classes, especially in light of the comparison with 2D-LPP, whose three-dimensional projection is shown in Fig. 5(b). At the same time, the images of the same class locate very closely in 2D-FDLPP subspace. The result illustrates that 2D-FDLPP is able to minimizing the within-class distance, while maximizing the between-class distance. Therefore, 2D-FDLPP has more discriminating power than that of 2D-LPP. 


\subsection{Comparison between 2D-FDLPP and 2D-LDA}

2D-LDA attempts to seek a set of optimal discriminating vectors to form a transform image matrix $X_{i}$ by maximizing the 2D Fisher criterion denoted as

$$
\begin{aligned}
J_{2 D L D A} & =\frac{\operatorname{tr}\left(T S_{B}\right)}{\operatorname{tr}\left(T S_{W}\right)} \\
& =\frac{A^{T}\left(\sum_{i=1}^{c} n_{i}\left(\bar{X}^{(i)}-\bar{X}\right)^{T}\left(\bar{X}^{(i)}-\bar{X}\right)\right) A}{A^{T}\left(\sum_{i=1}^{c} \sum_{k=1}^{n_{i}}\left(X_{k}-\bar{X}^{(i)}\right)^{T}\left(X_{k}-\bar{X}^{(i)}\right)\right) A}
\end{aligned}
$$

where $\bar{X}^{(i)}$ is the mean matrix of the $i$ th class, $\bar{X}$ is the mean matrix of all the samples, and $X_{k}$ denotes the $k$ th sample.

From Eq. (10) it can be seen that the goal of 2D-LDA is to extract features that well discriminate a set of data that belongs to a number of classes, making samples from different classes far as much as possible from each other, while samples from same classes close as much as possible. Both 2DFDLPP and 2D-LDA are supervised algorithm and attend to preserve the discriminant information of samples. While the improvement of 2D-FDLPP algorithm over 2D-LDA algorithm benefits mostly from two aspects: (1) 2D-FDLPP introduced fuzzy assignment method to construct the weight matrix, so that more robust data presentation is obtained; (2) 2D-LDA evaluates the separability in terms of the distance between class means or the distance between the sample and its class mean, whereas 2D-FDLPP evaluate the separability in terms of the distance between samples, which aims to preserve the neighbor relationship of samples; (3) in 2DLDA, the distribution of variation for any two samples in the same class is the same, which means that each sample has the same contribution to the classification, while 2DFDLPP uses similarity coefficient to represent the similarity between two samples from the same class. 2D-FDLPP can be viewed as a combination of the ideas of both 2D-LDA and 2D-LPP, with soft assignment method to construct the similarity coefficients.

\section{Experimental Results}

In this section, we investigate the performance of our proposed 2D-FDLPP method for image recognition problems. We begin with handwritten examples to verify the classification power of 2D-FDLPP, and deal with the facial expression recognition task on Cohn-Kanade facial expression database, JAFFE facial expression database, finally, performance of 2D-FDLPP on face recognition is verified on ORL face database.

\subsection{Handwritten Examples}

In this section, the application of 2D-FDLPP in handwritten recognition will be discussed. The dataset used in our experiment is the MNIST handwritten digit data set [26], containing $28 \times 28$ pixel image of digits 0 to 9 . Some of the examples of MNIST dataset are shown in Fig. 6. In our

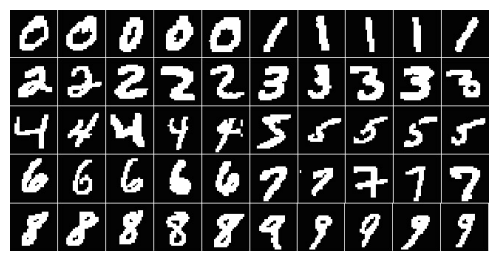

Fig. 6 Digit image samples from MNIST database.

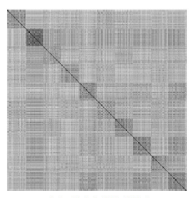

(a) 2D-FDLPP

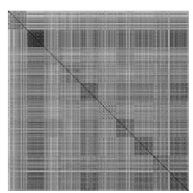

(b) 2D-LPP

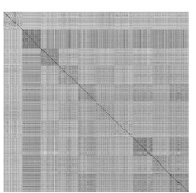

(c) 2D-LDA

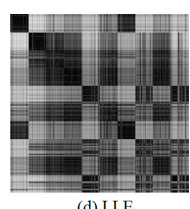

(d) LLE
Fig. 7 Euclidean distances between testing samples after different projections.

experiment, we verify the classification power of our proposed 2D-FDLPP method on the handwritten digits. First, we select 100 samples per class from the training set, and 500 samples per class from the testing set. The training set is used to obtain the optimal solution of Eq. (9) with fuzzy weight matrix, and then testing samples are embedded to the subspace by the transformation matrix.

To illustrate the discriminant power of 2D-FDLPP separating different classes and clustering the samples in the same class, we show the Euclidean distance between projected testing samples. Let $Y^{i}$ and $Y^{j}$ denote two testing samples embedded to the obtained subspace, the Euclidean distance between them are calculated as: $\sum_{k, l}\left(Y_{k, l}^{i}-Y_{k, l}^{j}\right)$.

The distributions of the distances between samples are shown in Fig. 7, and the result of 2D-FDLPP is compared with that of 2D-LPP, 2D-LDA and LLE [27]. LLE method is nonlinear dimensionality reduction method based on image vector. We compare the classification performance of 2D-FDLPP algorithm to that of 2D-LPP, 2D-LDA and onedimensional nonlinear method. It is clear that 2D-FDLPP has the block structure which means that it separates the ten digital classes successfully and makes the samples of the same class compact. For 2D-LPP, the illumination shows that the original neighboring samples are still close after projection. However, the samples of class 2, 3, 5, 8 are confused with samples of other classes. For 2D-LDA, 2, $3,4,5$ are separated clearly, 7 is well separated from other digits, while others are confused. For LLE, some of the digital classes are separated clearly (e.g. 0, 3, 5), while other classes are confused significantly.

\subsection{Facial Expression Recognition}

In this section, we discuss how 2D-FDLPP learns the fuzzy discriminant locality preserving subspace and its performances of facial expression recognition for six basic facial expressions (namely, anger, disgust, fear, happiness, sadness and surprise). The experiments are carried out on the widely used Cohn-Kanade facial expression database [28] 


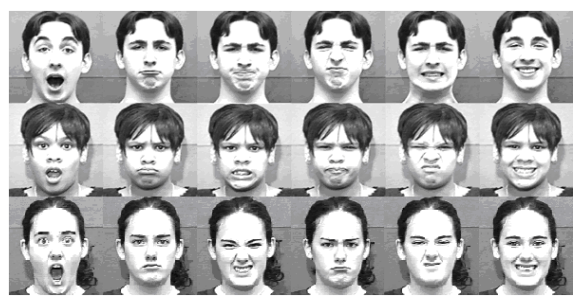

Fig. 8 Facial expression images from Cohn-Kanade database.

and JAFFE facial expression database [29]. The classifier design is not our emphasis. Therefore, we take nearest neighbor classifier for simplicity.

\subsubsection{Cohn-Kanade Facial Expression Database}

The Cohn-Kanade facial expression database consists of video sequences of subjects displaying distinct facial expressions, starting from neutral expression and ending with the peak of the expression. As some subjects in CohnKanade database show less than six facial expressions, we use a subset with thirty subjects for our experiments. For each expression of a subject, the last eight frames in the videos are selected, and we treat these frames as static images for both training and testing. The images are manually cropped to a central face image containing the main facial components (as eyebrows, eyes, nose and chin) and resized to $120 \times 120$. The uniform background was eliminated. Some of the samples in Cohn-Kanade database are shown in Fig. 8.

In the classical facial expression recognition, the original data are split in two disjoint parts, training and testing data sets. To form the training set, we randomly select different training samples $p(p=1,2,3)$ from each subject of each facial expression (i.e. the number of training samples of each class is $p \times 30$ ), while the rest of the images are used for testing.

We compare the proposed 2D-FDLPP algorithm with other matrix-based dimensional reduction methods, i.e. 2DLPP, 2D-LDA and 2D-PCA. For each $p$, the recognition accuracies of algorithm with varied dimensions are reported and shown in Fig. 9. Furthermore, Table 1 reports the highest recognition accuracies obtained by the six algorithms. It can be seen from the illustrations that 2D-FDLPP algorithm outperforms other algorithms significantly, and it achieves the highest recognition rates among the comparing algorithms. It shows that 2D-FDLPP has more discriminating ability to separate different pattern classes and makes samples in the same class more compact. Therefore, 2D-FDLPP obtains better result in facial expression recognition.

\subsubsection{JAFFE Facial Expression Database}

The JAFFE facial expression database consists of $213 \mathrm{im}-$ ages of Japanese female facial expressions. Ten subjects posed three or four examples for each of the six basic facial expressions plus neural face. First, the facial images are

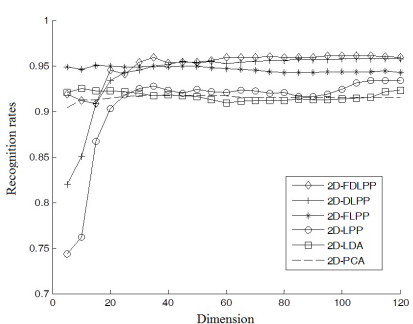

(a)

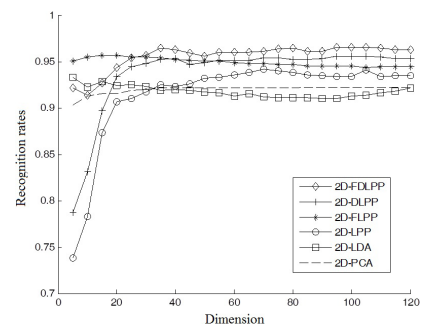

(b)

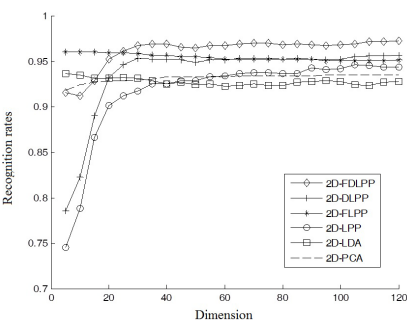

(c)

Fig. 9 Recognition accuracy versus dimensionality reduction on the Cohn-Kanade facial expression database (a) $p=1$; (b) $p=2$; (c) $p=3$.

Table 1 Comparison of the highest recognition accuracies (\%) of 2DFDLPP, 2D-LPP, 2D-LDA, and 2D-PCA algorithm on Cohn-Kanade facial expression database.

\begin{tabular}{|c|c|c|c|}
\hline & case 1 & case 2 & case 3 \\
\hline 2D-FDLPP & $95.9(120 \times 90)$ & $96.5(120 \times 95)$ & $97.2(120 \times 120)$ \\
\hline 2D-DLPP & $95.5(120 \times 85)$ & $95.6(120 \times 95)$ & $95.9(120 \times 120)$ \\
\hline 2D-FLPP & $95.2(120 \times 15)$ & $95.6(120 \times 15)$ & $96.0(120 \times 5)$ \\
\hline 2D-LPP & $93.4(120 \times 110)$ & $94.1(120$ & $94.6(120 \times 105)$ \\
\hline 2D-LDA & 92.5( & $20 \times 5)$ & $93.7(120 \times 5)$ \\
\hline 2D-PCA & $91.7(120 \times 30)$ & $92.2(120 \times 85)$ & $93.5(120 \times 95)$ \\
\hline
\end{tabular}

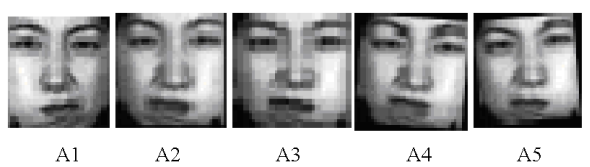

Fig. 10 Synthetic facial expression images on JAFFE database.

manually cropped and resized to $32 \times 32$. The total number of facial expression images in JAFFE database is limited, and it contains only frontal face images. To verify the robustness of 2D-FDLPP algorithm, we extended the database by synthetizing various facial images according to the following methods:

For original facial image A:

(1) Make mirror symmetry transform to A, to get A1;

(2) Scale A by a coefficient of 0.9 , to get A2;

(3) Scale A by a coefficient of 1.1, to get A3;

(4) Rotate A by an angle of 5degree (left), to get A4;

(5) Rotate A by an angle of 5 degree (right), to get A5;

The samples of synthetic facial expressions are shown in Fig. 10. In our experiment, six-fold cross validation method which is similar with that in [30] is conducted on the extended JAFFE database. The extended JAFFE facial expression database is equally divided into six subsets. Then one of the subsets is selected for testing while the remain- 


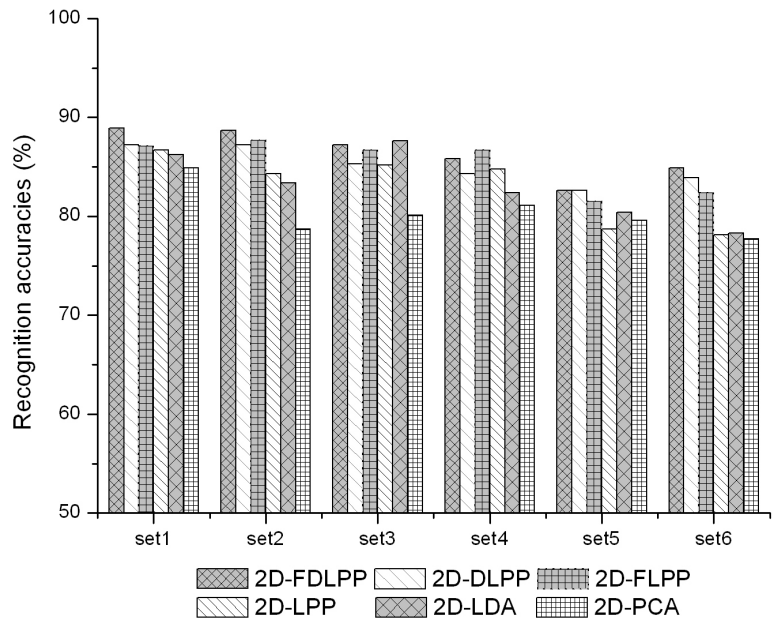

Fig. 11 Comparison of the highest recognition accuracies (\%) of 2DFDLPP, 2D-LPP, 2D-LDA and 2D-PCA algorithms on JAFFE facial expression database.

der is used to construct the training set. Experiments are repeated for six times, with different test subset being used for each time.

We compare the proposed 2D-FDLPP algorithm with 2D-LPP, 2D-LDA, and 2D-PCA. The highest recognition accuracies achieved for each experiment case is compared in Fig. 11. In short, the recognition performance of 2DFDLPP is better than all other tested dimensionality reduction algorithms in facial expression recognition. For subset 2 , the best recognition accuracies achieved by 2D-FDLPP is $88.7 \%$, while the best recognition accuracies obtained by 2D-LPP, 2D-LDA, 2D-PCA are $84.3 \%, 83.4 \%$, and 78.7\%, respectively. 2D-FDLPP algorithm gives comparable results by using the simplest nearest neighbor classifier, and it demonstrates the superiority of 2D-FDLPP algorithm.

\subsection{Face Recognition}

In this section, the proposed 2D-FDLPP method is used for face recognition and tested on Olivetti-Oracle Research Lab (ORL, http://www.cam-orl.co.uk, [31]). The ORL face database contains images from 40 individuals, each providing 10 different images. The images are varied in pose, illumination and facial expression. In our experiment, the images are manually cropped and resized to $48 \times 48$ pixels and no other preprocessing is conducted. Some example images from the ORL face database are shown in Fig. 12.

In our experiment, some images are randomly selected for training, while the remaining is used for testing. Furthermore, a series of test are designed to compare the performance of 2D-FDLPP to that of 2D-LPP, 2D-LDA, and 2D-PCA methods under conditions where the sample size is varied. More specifically, we use 2, 3, 4, and 5 image samples randomly per class for training and the remaining samples for testing. Figure 13 presents the highest recognition accuracies of the six methods for four experiments. This figure indicates that the performance of 2D-FDLPP is

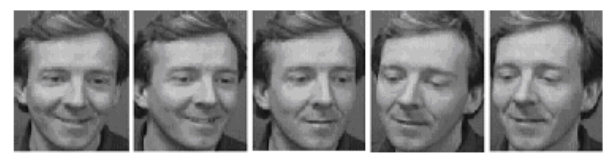

Fig. 12 Face images from the ORL face databases.

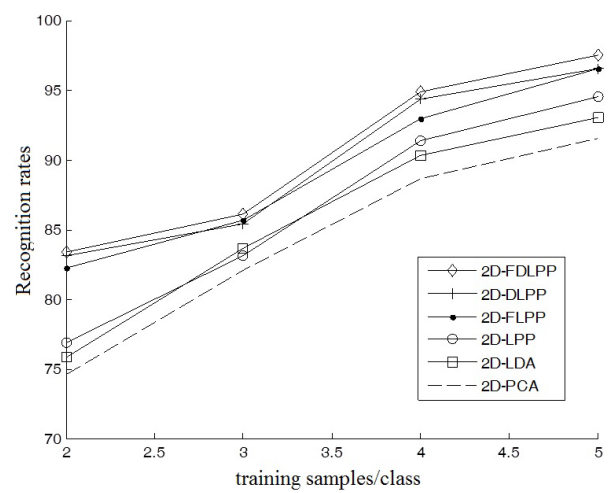

Fig. 13 Recognition accuracy for different training samples on the ORL face database.

Table 2 The average CPU time (s) consumed for training and testing, the highest recognition accuracies (\%) and the corresponding dimensions of the six two-dimensional methods (CPU: Intel Core2 2.83GHz, RAM: 3.50GB).

\begin{tabular}{lllllll}
\hline Method & 2D-PCA & 2D-LDA & 2D-LPP & 2D-FLPP & 2D-DLPP & 2D-FDLPP \\
\hline $\begin{array}{l}\text { Recognition } \\
\text { accuracy }\end{array}$ & 88.6 & 90.3 & 91.4 & 92.9 & 94.3 & 95.0 \\
$\begin{array}{l}\text { Dimension } \\
\text { CPU time }\end{array}$ & $48 \times 4$ & $48 \times 4$ & $48 \times 4$ & $48 \times 6$ & $48 \times 42$ & $48 \times 14$ \\
\hline
\end{tabular}

better than that of other tested algorithms.

In addition, the average CPU time consumed for training and testing, the recognition accuracy and the corresponding dimensions of the six algorithms are given in Table 2 (All the face images were imported from .mat, which is the data storing format in Matlab). 2D-FDLPP algorithm achieves the maximal recognition rate of $95 \%$ using 14 features, and the consumed CPU time is slightly higher than that of 2D-DLPP and 2D-FLPP.

\section{Conclusions}

In this paper, we proposed a novel subspace learning algorithm which has fuzzy discriminant locality preserving ability. Motivated by the advantage of fuzzy set, we utilize the fuzzy assignment to generate fuzzy weight matrix to denote the relationship between samples. Moreover, discriminant objective function is proposed to improve the discriminating power of the algorithm. Experiments are conducted on handwritten digital images, facial expression images, and face images. Experimental results demonstrate that 2DFDLPP algorithm can achieve encouraging results in pattern recognition tasks. 


\section{Acknowledgments}

This work is supported by the National Natural Science Foundation of China (No. 31201358). The authors gratefully acknowledge the helpful comments and suggestions of the reviewers, which have improve the presentation.

\section{References}

[1] S. Kong, J. Heo, B. Abidi, J. Paik, and M. Abidi, "Recent advances in visual and infrared face recognition - A review," Comput. Vis. Image Understand., vol.97, pp.103-155, 2005.

[2] B. Fasel and J. Luettin, "Automatic facial expression: A survey," Pattern Recognit., vol.36, pp.259-275, 2003.

[3] M. Liang, M. Sun, K.M. Carter, A.O. Hero III, and S. Savarese, "Unsupervised object pose classification from short video sequences," British Machine Vision Conference (BMVC), Sept. 2009.

[4] P.Y. Simard, Y.A. LeCun, J.S. Denker, and B. Victorri, "Transformation invariance in pattern recognition - Tangent distance and tangent propagation," Lect. Notes Comput. Sci., vol.1524, pp.549-550, 1998.

[5] R. Rao and D. Rudeman, "Learning lie groups for invariant visual perception," Advances in Neural Information Processing Systems (NIPS), 1998.

[6] J. Winn, A. Criminist, and T. Minka, "Object categorization by learned universal visual dictionary," Proc. IEEE International Conference on Computer Vision (ICCV), vol.2, pp.1800-1807, 2005.

[7] D. Donoho, "High-dimensional data analysis: The curses and blessings of dimensionality," AMS Math Challenges Lecture, 2000.

[8] W.S. Yambor, "Analysis of PCA-based and Fisher Discriminantbased Image Recognition Algorithms," Computer Science Technical Report, July 2000.

[9] P.N. Belhumeur, J.P. Hespanha, and D.J. Kriegman, "Eigenfaces vs. Fisherfaces: Recognition using class specific linear projection," IEEE Trans. Pattern Anal. Mach. Intell., vol.19, pp.711-720, 1997.

[10] X. He, S. Yan, Y. Hu, P. Niyogi, and H. Zhang, "Face recognition using Laplacianfaces," IEEE Trans. Pattern Anal. Mach. Intell., vol.27, pp.328-340, 2006.

[11] J.C. Isaacs, S.Y. Foo, and A.M. Baese, "Novel kernels and kernel PCA for pattern recognition," International Symposium on Computational Intelligence in Robotics and Automation, Jacksonville, 2007.

[12] C. Shan, S. Gong, and P.W. McOwan, "A comprehensive empirical study on linear subspace methods for facial expression analysis," Proc. Conference on Computer Vision and Pattern Recognition Workshop (CVPRW), 2006.

[13] F. Wang, J. Wang, C. Zhang, and J. Kwok, "Face recognition using spectral features," Pattern Recognit., vol.40, pp.2786-2797, 2007.

[14] J. Yang, D. Zhang, A.F. Frangi, and J. Yang, "Two-dimensional PCA: A new approach to appearance-based face representation and recognition," IEEE Trans. Pattern Anal. Mach. Intell., vol.26, pp.151-157, 2004.

[15] L. Wang, X. Wang, and J. Feng, "On image matrix based feature extraction algorithms," IEEE Trans. Syst., Man Cybern., B, vol.36, pp.195-197, 2006.

[16] D. Hu, G. Feng, and Z. Zhou, "Two-dimensional locality preserving projections (2DLPP) with its application to palmprint recognition," Pattern Recognit., vol.40, pp.339-342, 2007.

[17] X. He and P. Niyogi, "Locality preserving projections," in Advances in Neural Information Processing Systems, MIT Press, Cambridge, 2003.

[18] R. Zhi and Q. Ruan, "Facial expression recognition based on twodimensional discriminant localiry preserving projections," Neurocomputing, vol.71, pp.1730-1734, 2008.
[19] S. Chen, H. Zhao, M. Kong, and B. Luo, "2D-LPP: A twodimensional extension of locality preserving projections," Neurocomputing, vol.70, pp.912-921, 2007.

[20] D. Cai, X. He, and J. Han, "Using Graph Model for Face Analysis," Technical Report, Department of Computer Science, University of Illinois at Urbana-Champaign, 2005.

[21] D. Cai, X. He, and J. Han, "Spectral regression for dimensionality reduction," Technical Report, Department of Computer Science, University of Illinois at Urbana-Champaign, 2007.

[22] D. Cai, X. He, and J. Han, "Spectral regression: A unified subspace learning framework for content-based image retrieval," ACM Multimedia, Augsburg, Germany, 2007.

[23] Y. Xu, G. Feng, and Y. Zhao, "One improvement to two-dimensional locality preserving projection method for use with face recognition," Neurocomputing, vol.73, pp.245-259, 2009.

[24] M. Keller, M.R. Gray, and J.A. Givens, "A fuzzy k-nearest neighbor algorithm," IEEE Trans. Syst. Man Cybern., vol.15, pp.580-585, 1985.

[25] R. Zhi, Q. Ruan, and J. Wu, "Dual two-dimensional fuzzy class preserving projection for facial expression recognition," IEICE Trans. Inf. \& Syst., vol.E91-D, no.12, pp.2880-2883, Dec. 2008.

[26] http://yann.lecun.com/exdb/mnist/

[27] S.T. Roweis and L.K. Saul, "Nonlinear dimensionality reduction by locally linear embedding," Science, vol.290, pp.2323-2326, 2000.

[28] T. Kanade, J. Cohn, and Y. Tian, "Comprehensive database for facial expression analysis," Proc. International Conference on Automatic Face and Gesture Recognition (FGR), Grenoble, France, 2000.

[29] M. Lyons, S. Akamatsu, M. Kamachi, and J. Gyoba, "Coding facial expressions with Gabor wavelets," Proc. Third IEEE Conference on Face and Gesture Recognition, Nara, Japan, 1998.

[30] Z. Zhang, M.J. Schuster, and S. Akamatsu, "Compparison between geometry-based and Gabor-wavelet based facial expression recognition using multi-layer perception," Proc. IEEE 3rd International Conference on AFGR, 1998.

[31] F. Samaria and A. Harter, "Parameterisation of a stockastic modell for human face identification," Second IEEE Workshop on Applications of Computer Vision, 1994.

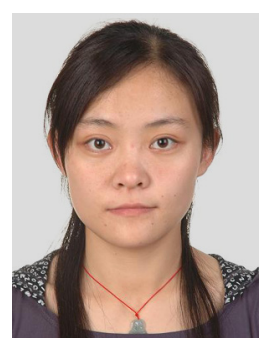

Ruicong Zhi received the Ph.D degree in Signal and Information Processing from Beijing Jiaotong University, Beijing, China, in 2010, the B. S. degree in biomedical engineering from Beijing Jiaotong University, Beijing, China, in 2005. From 2008 to 2009, she visited the Sound and Image Processing Laboratory, Royal Institute of Technology (KTH), Stockholm, Sweden, as a joint Ph.D student. She is currently an associate researcher in China National Institute of Standardization, Beijing, China. She has published more than 30 papers. Her research interests included sensory metrology, consumer test, and pattern recognition. She has been the recipient of several awards, including the Award for Best Ph.D Thesis of Beijing Jiaotong University. 


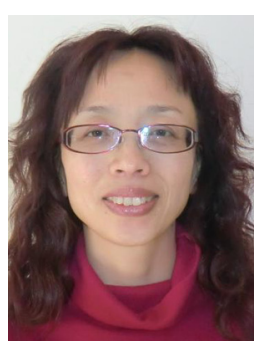

Lei Zhao received her Ph.D. degree in 2004 from the College of Food Science and Nutritional Engineering of China Agricultural University, Beijing, China. She did the postdoctoral research work then. In 2006, she worked at Food and Agriculture Standardization Institute of China National Institute of Standardization, and held the posts of the deputy director of Food and Agriculture Standardization Institute and the chief of Food Sensory Analysis Laboratory successively. At present she is the person in charge of food sensory analysis research field. She has coauthored over 50 articles, 6 books and 12 China national standards. She received the first and second prize for Science and Technology of China General Chamber of Commerce in 2010 and 2012 respectively, the Technological Innovation Prize - Technological Progress First Prize of Chinese Institute of Food Science and Technology in 2012. She is the person in charge of internal corresponding technical work of Sensory Analysis Sub-committee of Food Technical Committee of International Organization for Standardization (ISO/TC34/SC12), the member of Committee E18 on Sensory Evaluation of Materials and Products of American Society for Testing and Materials (ASTM) (ASTM/E18), and the member of Sensory Scientist Society (SSP).

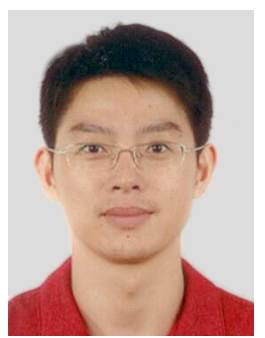

Bolin Shi received the Ph.D degree in food science from China Agriculture University, Beijing, China, in 2008, the M.S. degree in food science from China Agriculture University, Beijing, China, in 2005, and the B.S. degree in food science from China Agriculture University, Beijing, China, in 2003. He was a postdoctor in Shanghai University from 2008 to 2010 , focusing on intelligent sensory analysis. He is currently an associate researcher in China National Institute of Standardization. He has published more than 30 papers, and also has a national patent. His main research interests include sensory evaluation and intelligent sensory analysis.

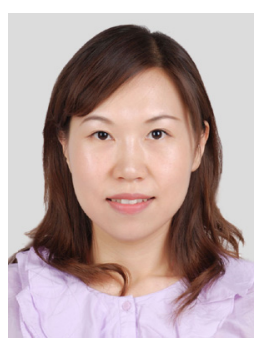

Yi Jin was born in Heibei, China, in1982 and received the Ph.D degree in Signal and Information Processing from Beijing Jiaotong University, China, in2010. She is currently an assistant professor in the School of Computer Science and Information Technology, Beijing Jiaotong University. Her research interests include image processing, pattern recognition, computer vision and machine learning. 